\title{
A novel system analysis methodology: transform method, relation spectrum, and system filter
}

\author{
Gang Liu and Jing Wang
}

\begin{abstract}
We have presented a controllable and human-readable polynomial neural network (CR-PNN) that is the first human-readable neural network. One can imagine its influence on system identification. Subsequently, we developed a relation spectrum in a medical application, which is likely to stand alongside the Fourier spectrum. However, the system analysis methodology is incomplete in contrast to signal processing methodology. Here, we presented the system filters for the first time. In this paper, we used the simulation system to verify the availability of the system analysis methodology. The system analysis methodology showed great properties in system identification and filter. The contribution of this paper is the system analysis methodology: transform method (CR-PNN), relation spectrum, and system filter design.
\end{abstract}

(We're strong believers in Open Source, and provide system analysis methodology code for others.

GitHub: https://github.com/liugang1234567/CR-PNN\#cr-pnn)

Index Terms-CR-PNN, relation Spectrum, system identification, system filters, transfer function

\section{INTRODUCTION}

For years, system parameter identification is a classic problem in signal modeling. Concretely, it is basic for system analysis and control design $[1,2]$ and become a hot issue in the field of control and signal modeling $[3,4]$. A neural network $(\mathrm{NN})$ is an excellent "toy" that can learn a complex, nonlinear function [5-7]. However, unfortunately, over the years, NN is often ineffective for system identification due to its "black box" nature. Lately, Gang Liu et al., the authors of the system analysis methodology, presented a Human-Readable NN, called CR-PNN, which may compensate this regret [8].

For centuries, Fourier spectrum has crucial applications in various fields. In essence, Fourier spectrum is the amplitudes of trigonometric function decomposed from the original function. Do you remember the other famous decompose method, Taylor expansion? Taylor expansion decomposes original function to a series of polynomials. However, Taylor expansion has been still "sleeping" in a math book. Here, we try to "wake up" it and constitute a polynomial spectrum or relation spectrum that represents the relationship of input and output.

A filter is a tool used to remove undesired components and remain needed components. In previous studies, the filter is usually used for signal processing. Here, we show the filter to use for removing the functional components in the system for the first time.

Gang Liu is a doctoral student of Institute of Robotics and Intelligent Systems, School of Mechanical Engineering, Xi'an Jiao Tong University, Xi'an 710049 China (e-mail: gangliu.6677@gmail.com) [Put forward this system analysis methodology].

Jing Wang is an associate professor of Institute of Robotics and Intelligent Systems, School of Mechanical Engineering, Xi'an Jiao Tong University, Xi'an 710049 China (e-mail: wangpele@gmail.com) [Offer guidance to this study].
This methodology offers a fresh perspective on system analysis, and the system analysis methodology consists of three main parts: transform method (CR-PNN), relation spectrum, and system filter.

\section{The Process Of Methodology}

The process is shown as follows:

1. For an unknown system, we should use CR-PNN to simulate the real system.

2. Translate the CR-PNN model into the relation spectrum.

3. Design system filter.

Measured system (Matlab code):

$$
y=x_{1}+0 x_{2}-2 x_{3}+4 x_{4}^{2}-5 x_{5} x_{4}+8 x_{6}{ }^{2}-x_{7} x_{4}+0 x_{8}
$$

Input:

Input Matlab code

\begin{tabular}{cc}
\hline$x_{1}$ & $\mathrm{I}(:, 1)=\sin (1.5 * \mathrm{t}) ;$ \\
$x_{2}$ & $\mathrm{I}(:, 2)=\sin \left(1 / 2 *_{\mathrm{t}+60) ;}\right.$ \\
$x_{3}$ & $\mathrm{I}(:, 3)=\operatorname{sawtooth}(2 *, 4)=\sin \left(5 *_{\mathrm{t}+20) ;}\right) ;$ \\
$x_{4}$ & $\mathrm{I}(:, 5)=\operatorname{sawtooth}\left(1 / 4 *_{\mathrm{t}+100) ;}\right.$ \\
$x_{5}$ & $\mathrm{I}(:, 6)=\sin \left(2 *_{\mathrm{t}+20) ;}\right.$ \\
$x_{6}$ & $\mathrm{I}(:, 7)=\sin \left(3 *_{\mathrm{t}+34) ;}\right.$ \\
$x_{7}$ & $\mathrm{I}(:, 8)=\sin \left(1 / 9 *_{\mathrm{t}+25) ;}\right.$ \\
$x_{8}$ &
\end{tabular}

Output:

Output

Matlab code

$y \quad \mathrm{~S}(:, 1)=\mathrm{I}(:, 1)-2 * \mathrm{I}(:, 3)+4 * \mathrm{I}(:, 4) \wedge^{\wedge} 2-5 * \mathrm{I}(:, 5) *{ }^{*} \mathrm{I}(:, 4)+8 * \mathrm{I}$
$(:, 6) . \wedge 2-\mathrm{I}(:, 7) . * \mathrm{I}(:, 4)$

\section{How To USE CR-PNN}

Without a doubt, if we transform all real systems to Taylor expansion, we could identify the system parameters.

CR-PNN is the tool to achieve this transformation [8]. CR-PNN is the Taylor expansion in the form of a network. The number of layers represents precision. In the literature, we imitate derivatives in Taylor expansion by the error back-propagation algorithm. Coefficients of Taylor expansion are stored in the weight $W$ of the network.

Here, as an example, we show a system that contains 8 inputs and 1 output in figure 1 . $L$ represents the pre-estimated degree of the system. We recommend approximating the real system form a low degree to a high degree. Additionally, the input should be normalized into $[-1,1]$, the output had better be normalized into $[-1,1]$.

The result of the approximation of the Measured system is shown in figure 2 . 


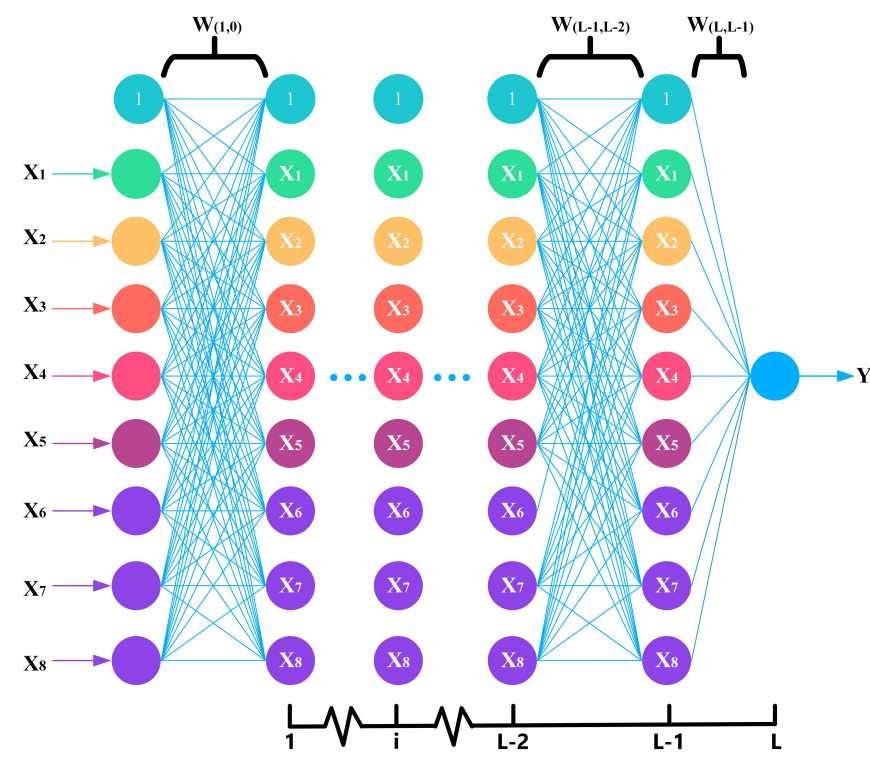

Fig. 1. Transform method (CR-PNN).

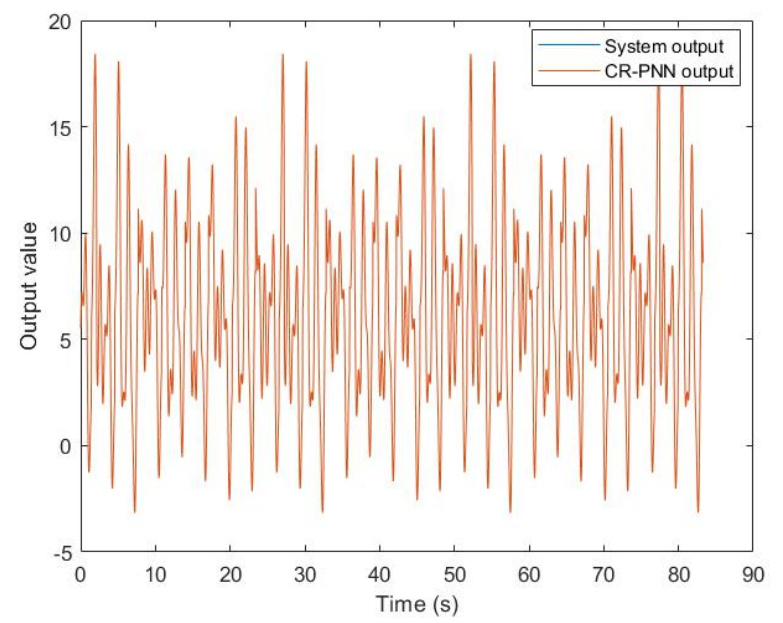

Fig. 2. Result of approximation using CR-PNN with $\mathrm{L}=3$.

\section{How To Use Relation Spectrum}

Because the computational complexity of CR-PNN is very low, we could generate the relation spectrum (Our code: Read_PNN).

Two explanations about the relation spectrum are shown as follows:

(1) The relation spectrum expresses the impact to output variable from the input variable, and the impact contains independent and interaction effects in different orders.

(2) It is widely known that every functional relationship can be expressed by the accumulation of trigonometric function or polynomial. For the expression of a trigonometric function, the typical example is Fourier frequency spectrum. Here, the relation spectrum is the polynomial spectrum.

The relation spectrum for the Measured system is shown in figure 3 . Furthermore, the relation spectrum can be read by way of a checklist. "Position of items" corresponds to "Items" in Table I. [ In the application, we check the list by code. Example: Matlab code: $t(120)]$

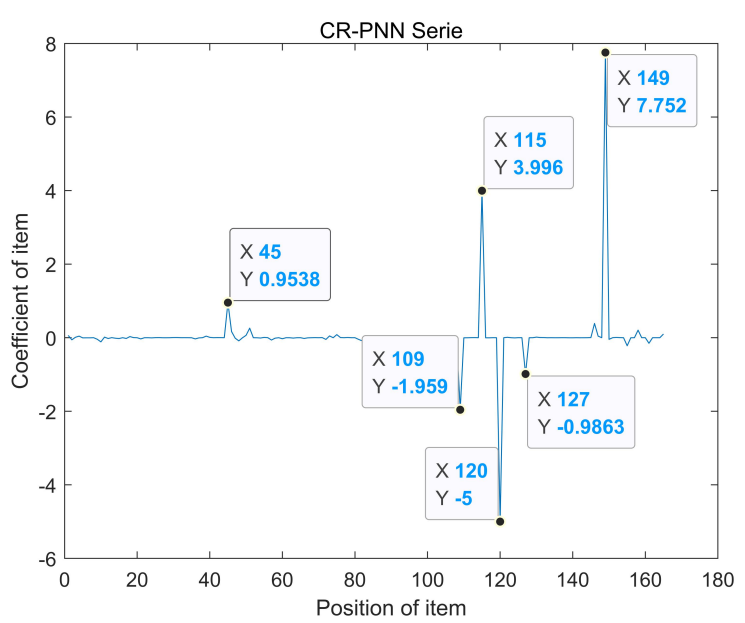

Fig. 3. Relation spectrum. The coefficient of item is almost equal to the Measured system. [ Errors may derive from the similarity of input.]

TABLE I

POSITION OF ITEM

\begin{tabular}{ccc}
\hline \hline Position & Item \\
\hline 1 & & $x_{1}^{3}$ \\
2 & $x_{1}^{2} x_{2}$ \\
3 & & $x_{1}{ }^{2} x_{3}$ \\
4 & $\ldots$ & $x_{1}{ }^{2} x_{4}$ \\
& & \\
45 & $\ldots$ & $x_{1}$ \\
109 & $\ldots$ & $x_{3}$ \\
115 & $\ldots$ & $x_{4}{ }^{2}$ \\
120 & $\ldots$ & $x_{4} x_{5}$ \\
127 & & $x_{4} x_{7}$ \\
149 & & $x_{6}{ }^{2}$ \\
\hline \hline
\end{tabular}

\section{How To Use System Filter}

Here, we show an example. Assumption: $x_{4} x_{7}$ is regarded as the noise. The CR-PNN model without noise is expressed as:

$$
y_{c r-p n n}=0.9538 x_{1}-1.959 x_{3}+3.996 x_{4}{ }^{2}-5 x_{4} x_{5}+7.752 x_{6}{ }^{2}
$$

Where $y_{c r-p n n}$ is the output of the CR-PNN model without noise.

\section{DISCUSSION AND CONCLUSION}

For more researchers in different areas to understand and use this system analysis methodology, the paper is very short. However, we demonstrated the properties of the methodology by a lucid measured system. 
1) CR-PNN has shown outstanding approximation properties in figure 2 .

2) The relation spectrum reproduced the measured system and showed excellent analysis properties (see Fig.3 and TABLE I).

3) The system filter is feasible.

The novel system analysis methodology may benefit researchers in many fields, especially for researchers in the signal modeling and system analysis.

\section{REFERENCES}

[1] N. Li, S. J. Guo, and Y. Q. Wang, "Weighted preliminary-summation-based principal component analysis for non-Gaussian processes," Control Engineering Practice, vol. 87, pp. 122-132, Jun, 2019.

[2] J. X. Ma, W. L. Xiong, J. Chen, and F. Ding, "Hierarchical identification for multivariate Hammerstein systems by using the modified Kalman filter," Iet Control Theory and Applications, vol. 11, no. 6, pp. 857-869, Apr, 2017.

[3] P. Ma, and F. Ding, "New gradient based identification methods for multivariate pseudo-linear systems using the multi-innovation and the data filtering," Journal of the Franklin Institute-Engineering and Applied Mathematics, vol. 354, no. 3, pp. 1568-1583, Feb, 2017.

[4] S. Y. Liu, F. Ding, L. Xu, and T. Hayat, "Hierarchical Principle-Based Iterative Parameter Estimation Algorithm for Dual-Frequency Signals," Circuits Systems and Signal Processing, vol. 38, no. 7, pp. 3251-3268, Jul, 2019.

[5] S. C. Gao, M. C. Zhou, Y. R. Wang, J. J. Cheng, H. Yachi, and J. H. Wang, "Dendritic Neuron Model With Effective Learning Algorithms for Classification, Approximation, and Prediction," Ieee Transactions on Neural Networks and Learning Systems, vol. 30, no. 2, pp. 601-614, Feb, 2019.

[6] Y. P. Pan, and H. Y. Yu, "Biomimetic Hybrid Feedback Feedforward Neural-Network Learning Control," Ieee Transactions on Neural Networks and Learning Systems, vol. 28, no. 6, pp. 1481-1487, Jun, 2017.

[7] S. Li, H. Q. Wang, and M. U. Rafique, "A Novel Recurrent Neural Network for Manipulator Control With Improved Noise Tolerance," Ieee Transactions on Neural Networks and Learning Systems, vol. 29, no. 5, pp. 1908-1918, May, 2018.

[8] G. Liu, and J. Wang, "A Polynomial Neural Network with Controllable Precision and Human-Readable Topology for Prediction and System Identification," arXiv e-prints, https://ui.adsabs.harvard.edu/abs/2020arXiv200403955L, [April 01, 2020, 2020]. 